Published in final edited form as:

Handb Clin Neurol. 2013 ; 110: 283-294. doi:10.1016/B978-0-444-52901-5.00023-X.

\title{
Rehabilitation robotics
}

\author{
H.I. KREBS ${ }^{1,2,{ }^{*}}$ and B.T. VOLPE ${ }^{3}$ \\ ${ }^{1}$ Department of Mechanical Engineering, Massachusetts Institute of Technology, Cambridge, MA, \\ USA \\ ${ }^{2}$ Department of Neurology and Division of Rehabilitative Medicine, University of Maryland School \\ of Medicine, Baltimore, MD, USA \\ ${ }^{3}$ Department of Neurology and Neuroscience, Weill Medical College of Cornell University, White \\ Plains, NY, USA
}

\section{Abstract}

This chapter focuses on rehabilitation robotics which can be used to augment the clinician's toolbox in order to deliver meaningful restorative therapy for an aging population, as well as on advances in orthotics to augment an individual's functional abilities beyond neurorestoration potential. The interest in rehabilitation robotics and orthotics is increasing steadily with marked growth in the last 10 years. This growth is understandable in view of the increased demand for caregivers and rehabilitation services escalating apace with the graying of the population. We will provide an overview on improving function in people with a weak limb due to a neurological disorder who cannot properly control it to interact with the environment (orthotics); we will then focus on tools to assist the clinician in promoting rehabilitation of an individual so that $\mathrm{s} / \mathrm{he}$ can interact with the environment unassisted (rehabilitation robotics). We will present a few clinical results occurring immediately poststroke as well as during the chronic phase that demonstrate superior gains for the upper extremity when employing rehabilitation robotics instead of usual care. These include the landmark VA-ROBOTICS multisite, randomized clinical study which demonstrates clinical gains for chronic stroke that go beyond usual care at no additional cost.

\section{INTRODUCTION}

There is a silent revolution in the making. Unbeknownst to most clinicians, large corporations are making "smart homes" and "service robotics" the focus of their 21 st century endeavors. Smart homes aim to create a multitude of automatic devices embedded in the homes to aid people with everyday activities. Service or welfare robotics aim to create a multitude of robotic devices to assist humans in their daily activities and enhance the ability of caregivers and family members to take care of their clients and loved ones. Within this class of robots, this chapter will focus on what are known as "rehabilitation robots," which can be used to augment the clinician's toolbox in order to deliver meaningful restorative

*Correspondence to: Hermano Igo Krebs, Ph.D., Principal Research Scientist \& Lecturer, Massachusetts Institute of Technology, Mechanical Engineering Dept, 77 Massachusetts Ave, 3-137, Cambridge, MA 02139, USA. Tel: +1-617-253-8112, Fax: +1-617-258-7018, hikrebs@ mit.edu. 
therapy for an aging population. Rehabilitation robotics can support and enhance clinicians' productivity and effectiveness as they try to facilitate the individual's recovery.

This interest is inevitable: the demand for caregivers and rehabilitation services are growing apace with the aging population. By 2050 the contingent of seniors in the USA is expected to double from $13.3 \%$ of the population or 40 million to 80 million. With this growth comes an increased incidence of age-related maladies and disease including stroke. There are 795000 patients with new strokes every year, and the American Heart Association estimates that Americans will pay about $\$ 73.7$ billion in 2010 for stroke-related medical costs and disability (American Heart Association, 2010). The numbers for Europe paint a comparably bleak incidence rate, with over 1.1 million patients with new strokes every year. The Pacific Rim countries face yet greater challenges. The percentage of the Japanese population aged 65 and older is projected to increase to $30 \%$ by 2025 and 36\% by 2050 (Fig. 23.1). This situation creates an urgent need for new approaches to improve the effectiveness and efficiency of rehabilitation. It also creates an unprecedented opportunity to deploy technologies such as robotics to assist in the recovery process.

The goal of this chapter is to present a a brief overview of policies that are coalescing to make service robotics and smart homes the next "computer" revolution in this new century. We will offer a synopsis of advances in orthotics and then focus on the new advances in rehabilitation robotics that have moved the field beyond the "3Ds" - dirty, dangerous, distant - and that promise to revolutionize the way rehabilitation therapy is practiced.

\section{DIRTY, DANGEROUS, DISTANT}

Robotics is a relatively new engineering field. While historians debate its conception with examples present in ancient Greece, China, or Leonardo DaVinci's prolific work, it is generally accepted by "roboticists" that the first actual embodiment of the term, coined by Czech playwriter Karel Capek in the 1920s, took place in 1960 when Joseph Engelberger and George Devol delivered the Unimation robot to General Motors. The Institute of Electrical and Electronic Engineers (IEEE) put on a series of commemorative events marking this 50th anniversary (see, for example, the commemorative special issue: IEEE, 2010). In the past, robotics was applied usually when a task was too dirty, dangerous, or distant. Examples include application in industry and defense to improve productivity or to perform tasks too dangerous for humans.

However, there is also a need for robotic devices that require close proximity or contact with humans; this is markedly different from devices which often do not meet a human interactive safety standard. Great strides have been made in robot design and control that allow a class of robots to be used at home or in therapy gyms with an inherent risk no greater than that of an exercise treadmill.

\section{ACADEMIA, NATIONALINTEREST, ANDCORPORATION}

Figure 23.2 shows the number of citations for academic papers in journals searched with a typical search engine using the keywords: "rehabilitation robotics, service robotics, arm prosthesis, and smart home." In 1980, there were 16 papers on arm prosthesis with none 
employing the above keywords. The first robot deployed in clinical trials delivering rehabilitation therapy, the MIT-Manus, was developed in 1989. By the end of the 1990s, 76, 118,13 , and 55 papers, respectively, mentioned these four keywords. In recent years, the number of papers on rehabilitation robotics, service robotics, and smart home has shown a strong and sustained increase, with a small but noticeable growth in work on prosthesis. The magnitude of this change goes beyond the usual ebb-and-flow of activity in technologyrelated fields, and more change is predicted as corporate entities enter the arena.

Many dedicated researchers and companies are currently working in this field but, due to space limitations, only a few are described here. Large corporations like Philips are very interested in the smart home project and in rehabilitation robotics (see for example: http:// www.research.philips.com/technologies/index.html). Likewise, Honda and Toyota have been developing multiple robots aimed at the service and rehabilitation robotics market, and Microsoft is developing the next generation of OS (computer operational system), hoping to do for the field of smart home and robotics what it did for the computer industry. Bill Gates expressed his views on the potential of robotics:

Imagine being present at the birth of a new industry. It is an industry based on groundbreaking new technologies, wherein a handful of well-established corporations sell highly specialized devices for business use and a fast-growing number of start-up companies produce innovative toys, gadgets for hobbyists and other interesting niche products. .... . (like the computer industry) ... trends are now starting to converge and I can envision a future in which robotics devices will become a nearly ubiquitous part of our day-to-day lives. Technologies such as distributed computing, voice and visual recognition, and wireless broadband connectivity will open the door to a new generation of autonomous devices that enable computers to perform tasks in the physical world on our behalf. We may be on the verge of a new era, when the PC will get up off the desktop and allow us to see, hear, touch and manipulate objects in places where we are not physically present.

The Congressional Robotics Caucus comprising 37 members of Congress was formed "to recognize robotics technology as a pillar of 21st Century American innovation, highlight its growing importance in a wide variety of application areas, and emphasize its ability to inspire technology education." Human-robot interaction features prominently in all application areas identified in this "national roadmap" and is central to one of them: Medical and Healthcare Robotics (see, for example, US Congressional Robotics Caucus (0000) and National Science Foundation (0000)).

With so much exciting work in progress, we would be doing a disservice to the industry by attempting to cover the whole spectrum of robotic applications in a single chapter. While there is much to discuss in relation to smart homes and their potential benefits in a rehabilitation process which utilizes cabinets that lower to allow a person in a wheelchair to pick up a glass or monitors that remind a person to take his or her medication, we will focus here on emerging applications of robotics for more traditional rehabilitation. We will provide an overview on improving function in people with a weak limb due to a neurological disorder who cannot properly control it when interacting with the environment 
(orthotics), and then focus on tools to assist the clinician in promoting rehabilitation of an individual so that she/he can interact with the environment unassisted (rehabilitation robotics).

\section{ORTHOTICS}

Orthoses are passive or powered external devices for the neck, upper limb, trunk, and lower limb that are designed to guide motion, bear weight, align body structures, protect joints, or correct deformities. Unlike prostheses that replace a body part, orthoses are designed to work in cooperation with the intact body, and either control or assist movement (Krebs et al., 2006).

Common types of lower limb orthoses include foot orthosis (FO) shoe inserts for correcting ankle and foot deformities, ankle-foot orthoses (AFO) for correcting foot drop, functional knee orthoses (KO) for athletic injuries, hip abduction orthoses for limiting range of motion, long leg knee-ankle-foot orthosis (KAFO), and full length hip-knee-ankle-foot orthoses (HKAFO) for standing and gait stability. Trunk and neck orthoses include thoracolumbosacral orthoses (TLSO) for correcting scoliosis, lubrosacral orthoses (LSO) for stabilizing low back fractures, elastic trunk supports for preventing back injuries during lifting, and the common cervical orthoses (neck braces) for whiplash injuries or muscle spasms. Upper limb devices include shoulder and elbow slings for weight support during fracture healing, balanced forearm orthoses (BFO) for feeding assist, and an array of wrist, hand, and wrist-hand orthoses to position the joints or assist in activities of daily living. The fit of orthosis is critical as they must carry loads without interfering with normal skin and tissue function.

This section is not intended as an exhaustive review but it is intended to introduce powered orthoses that represent an exciting new field. We expect significant further developments in this area during the next decade but the premises and goals will not change.

\section{Orthotics for upper and lower limb joints}

There are several upper and lower limb orthoses being developed to actuate specific joints. They offer the promise of combining and blending assistive and rehabilitation technology into a single field. For example, it would allow community dwelling persons recovering from a stroke to perform functional tasks at home or anywhere with the concomitant rehabilitative potential. The promise is significant and has a large enough market potential to attract significant large players such as Honda. Hence, we foresee significant technological developments in the near future. While these devices enable individuals to perform functional tasks, much work remains to be done on how to apply this technology to therapeutics. For instance, present commercial devices have tepid evidence supporting their rehabilitative claims. The proof consists mainly of small open-label studies with no controls (Stein et al., 2007) or negative results showing no benefit as a rehabilitation tool when compared to usual care, as demonstrated by the lack of clinical evidence with the Bioness H200 in a randomized clinical trial (Weber et al., 2010). These initial results should be taken with the appropriate caveats. They indicate that much more research needs to be performed before we understand how to deploy these devices not only as assistive technology but also 
as rehabilitation tools. That said, we believe that the concept of determining the intent to move followed by that movement actually happening should be the basis of all assistive and therapeutic devices, as shown by the Myomo e100 that employs a surface EMG system to command its electrical actuator to flex or extend the elbow (Stein et al., 2007) or the Tybion that measures forces to command movement at the knee (see Fig. 23.3).

\section{Gait orthotics}

Typical objectives for walking orthotics are to establish stable weight bearing, to control the speed or direction of limb motion, and to reduce the energy required to ambulate. Several passive HKAFOs have been developed as paraplegic walking systems (Miller, 1997). Existing walking systems include the Hip Guidance Orthosis (HGO) that locks the knee joint but has freely moving hip and ankle joints (Major et al., 1981; Butler et al., 1984; Stallard et al., 1989) and the Reciprocating Gait Orthosis (RGO) that links opposite joints so that extension of the hip on one side leads to flexion on the contralateral side (Jefferson and Whittle, 1990). Although these systems can restore rudimentary gait for some people with spinal cord injury, the energy cost has resulted in limited use (Stallard et al., 1989; Whittle et al., 1991). For example, walking with HKAFOs and crutches requires five times the energy per meter of normal gait, while gait velocity is about one-third that of normal. Wheelchairs are a faster, more efficient means of travel, and therefore the device of choice.

Yet mechatronic HKAFOs hold substantial promise particularly in terms of reducing the energy expenditures of people. Two main approaches to provide a net power assistance to HKAFOs involve (a) functional electrical stimulation (FES) or (b) electrical motors. Several studies have shown improved gait speeds and lower energy consumption when FES and the RGO are combined (Solomonow et al., 1989, 1997; Hirokawa et al., 1990; Petrofsky and Smith, 1991; Isakov et al., 1992). Others have combined stimulation with the HGO (McClelland et al., 1987; Nene and Jennings, 1989); the Hybrid Assistive System (Popovic et al., 1989, 1990; Popovic, 1990), the Case Western Hybrid System (Ferguson et al., 1999; Marsolais et al., 2000), the Strathclyde Hybrid System) (Yang et al., 1997; Greene and Granat, 2003), and the Controlled-Brake Orthosis (Goldfarb and Durfee, 1996; Goldfarb et al., 2003).

Another approach to gait orthotics involves powered exoskeletons, which have a long and rich history starting with the first robotic arm built in the 1960s at Case Western Reserve University and the early powered walking machines pioneered by Tomovic and colleagues (Tomovic et al., 1973). Recent technological advances including embedded controllers, new materials, improved power supplies, and actuators have accelerated the development of such exoskeletons affording un-tether, low-mass, high cycle-life devices. For the first time, there are commercially viable advances in powered exoskeletons such as the Japanese "Hal" from Cyberdyne or the Israeli "Re-Walk" from Argo (Fig. 23.4). We will have to wait for the clinical outcomes research to understand energy expenditure of patients with spinal cord injury at or below T2, which we expect to be less than previous passive HKAFOs, and demonstration of stability of these devices when interacting with different environments (e.g., concrete or wood floors), slopes, and surfaces. That said, there is little doubt that for 
the first time there is a real alternative to wheelchairs, and we envision such patients using both alternatives depending on the situation.

\section{REHABILITATION ROBOTICS}

There is both a need and an opportunity to deploy technologies such as robotics to assist recovery. This, in essence, constitutes a paradigm shift moving the field of rehabilitation robotics beyond assistive technology (prosthetics and orthotics) that helps an individual cope with the environment to a new class of physically interactive, user-friendly robots that facilitate recovery. Therapeutic robots further a clinician's goal of facilitating recovery both by delivering measured therapy, and by affording new ways to evaluate patients' progress. There is no need to stress the importance. As previously noted, in the USA alone, there are 795000 patients with new strokes every year with over 6 million survivors. To put this clinical prevalence into perspective, 350000 persons have multiple sclerosis, 250000 have spinal cord injury, around 1 million people are affected by Parkinson disease, 1.7 million people are affected by a traumatic brain injury, and for every 1000 children born in the USA 2.8 have cerebral palsy (Krebs et al., 2008). Yet, while improvements in the acute care of stroke have been remarkable, the last 75 years of rehabilitation therapy practice and research have delivered few actual answers to ameliorate and maximize favorable outcomes in stroke survivors. We have essentially perpetuated long-time rehabilitation practices, many of which belong more in the realm of art and compassion than science. Hence, if the need is increasing, then current practices are unsatisfactory. However, during the 1970s and 1980s there was a paradigm shift in neuroscience: the concept that an adult brain was fixed and hardwired has proved to be false and we now understand that brain plasticity plays a significant role and offers a real window of opportunity to promote recovery (Jenkins and Merzenich, 1987; Hallett, 1999). That said, we still do not know how to maximize recovery. For the remainder of this chapter, we will focus exclusively on one approach that might enhance recovery: robots that support and improve the productivity of clinicians in their efforts to facilitate an individual's recovery. We will present a brief summary of a few rehabilitation robots for the upper and lower extremity, mention basic distinctions among robotic solutions, discuss the results of two meta-analyses for upper and lower extremity robotics, and finish by discussing two exciting examples for acute and chronic stroke studied by our research group. This overview is not intended to offer an exhaustive list of the many other researchers and companies involved in developing this area of robotics

\section{Different robots}

Figure 23.5 shows the Gym of Robots that we developed including the MIT-Manus for shoulder and elbow, anti-gravity, wrist, hand, anklebot, and skywalker robots. Figure 23.6 shows other commercial alternatives including the Osaka's University robot for shoulder and elbow, the bimanual Bimanutrac, the Amadeo, the Gait Trainer I, G-EO, and the Lokomat. Big picture

Two meta-analyses, one for the upper extremity and another for the lower extremity, are examined below. For the upper extremity, Kwakkel examined robotic training trials published up to October 2006 with the first generation of therapeutic robots (Kwakkel et al., 2008) (Fig. 23.7). A computerized literature search conducted in MEDLINE, CINAHL, 
EMBASE, Cochrane Controlled Trial Register, DARE, SciSearch, Doconline, and PEDro returned 173 hits. Only papers that compared robot training against a control group were included; studies comparing different forms of robotic therapy and studies on stroke comparing discharge values with admission values were excluded. The results demonstrated small but statistically significant improvements due to robot-assisted therapy, even when compared like-for-like with conventional therapy in stroke (Kwakkel et al., 2008). For the lower extremity, while several robotic devices already exist or are under development (e.g., MIT's Anklebot and Skywalker, KineAssist, Zero-G, Haptic Walker, UC Irvine's Pam and Pogo, Lopes, Motorika/Healthsouth Autoambulator), only the Gait Trainer I and the Lokomat have been used extensively with published reliable outcomes. The other devices, including our Anklebot, involve studies with less than 20 patients and will not be discussed here. In fact, studies with the Gait Trainer I and the Lokomat were the only ones included in a Cochrane Report (Mehrholz et al., 2007). This review demonstrated a small but substantial improvement favoring the robotic therapy group. In light of the negative results with chronic (Hornby et al., 2008) and subacute (Hidler et al., 2009) stroke patients, we re-examined the Cochrane report and found that most of the improvement reported was with the Gait Trainer I. The Lokomat, as employed until recently, imposed a fixed kinematic gait pattern determined from testing sessions with healthy subjects. In large studies for subacute and acute stroke, it was found that Lokomat training was inferior to usual care (Hornby et al., 2008; Hidler et al., 2009). We speculate that the Lokomat might not be affording the proper neurological sensorimotor inputs. Hornby et al. (2008) suggested that the Lokomat allows patients to remain completely passive and not actively engaged in the training session, resulting in unsuccessful gait therapy. Perhaps in view of these negative results, the Lokomat control scheme is being altered to afford an interactive experience, which in our opinion is essential in supraspinal neurological injuries.

\section{Subacute stroke}

Volpe and colleagues reported the composite results of robotic therapy with 96 consecutive subacute stroke inpatients admitted to Burke Rehabilitation Hospital in White Plains, NY (Volpe et al., 2001) (Fig. 23.8). All participants received conventional neurological rehabilitation during their participation in the study. The goal of the trial was to test whether movement therapy had a measurable impact on recovery. One group of patients was provided with as much movement therapy as possible to address a fundamental question: does goal-oriented movement therapy have a positive effect on neuromotor recovery after stroke?

Patients were randomly assigned to either an experimental (robot-trained) or control (robotexposure) group. Individuals in the robot-trained group were seen for five 1-hour sessions each week, and participated in at least 25 sessions of sensorimotor robotic therapy for the paretic arm. Patients were asked to perform goal-directed, planar reaching tasks that emphasized shoulder and elbow movements with their paretic arm. MIT-MANUS' low impedance guaranteed that the robot would not suppress attempts to move. When a patient could not move or deviated from the desired path or was unable to reach the target, the robot provided soft guidance and assistance dictated by an impedance controller (Krebs et al., 1998). This robot action (which we dubbed "sensorimotor" therapy) was similar to the 
"hand-over-hand" assistance that a therapist often provides during conventional therapy. It is interesting to note that this form of "assistance as needed," which has been a central feature of our approach from the outset (and a challenge for our robot designs), has recently been adopted and promoted by other groups (Reiner et al., 2005; Nef et al., 2007).

Individuals assigned to the robot-exposure (control) group were asked to perform the same planar reaching tasks as the robot therapy group. However, the robot did not actively assist the patient's movement attempts. When the subject was unable to reach towards a target, he or she could assist with the unimpaired arm. The robot supported the weight of the limb while offering negligible resistance to motion. For this control group, the task, the visual display, the audio environment (e.g., noise from the motor amplifiers), and the therapy context (e.g., the novelty of a technology-based treatment) were the same as for the experimental group. For ethical reasons (primarily to avoid discouraging them with excessive exposure to a task at which they did not succeed), patients in this group were seen for only 1 hour per week during their inpatient hospitalization.

The study was "double blinded" in that patients were not informed of their group assignment and therapists who evaluated their motor status did not know which group patients belonged to. Standard clinical evaluations included the upper extremity subtest of the Fugl-Meyer Assessment $(\mathrm{FM}$, maximum score $=66)$ from which we derived a Fugl-Meyer score for shoulder/elbow coordination (FM-SE, 42 out of 66); the MRC Motor Power Score for four shoulder and elbow movements (MP, maximum score $=20$ ); and the Motor Status Score which is divided into two subscales, one for shoulder and elbow movements (MS-SE, maximum score $=40$ ), and one for wrist and hand abilities (MS-WH, maximum score $=42$ ) (Aisen et al., 1997; Volpe et al., 1999, 2000; Krebs et al., 2000). The Fugl-Meyer test is a widely accepted measure of impairment in sensorimotor and functional grasp abilities. To complement the Fugl-Meyer scale, Burke Rehabilitation Hospital developed the Motor Status Scale to further quantify discrete and functional movements in the upper limb. The MS-SE and MS-WH scales expand the FM and have met standards for inter-rater reliability, significant intraclass correlation coefficients, and internal item consistency for inpatients (Fugl-Meyer et al., 1975).

Although the robot-exposure (control) and robot-treated (experimental) groups were comparable at admission based on sensory and motor evaluation and on clinical and demographic scales (enrollment into the study between 2 and 4 weeks poststroke), and both groups were inpatients in the same stroke recovery unit and received the same standard care and therapy for comparable lengths of stay, the robot-trained group demonstrated significantly greater motor improvement (higher mean interval change \pm SEM) than the control group on the MS-S/E and MP scores. In fact, the robot-trained group improved twice as much as the control group in these measures. Notably, these gains were specific to motions of the shoulder and elbow, the focus of the robot therapy. There were no significant between-group differences in the mean change scores for wrist and hand function, although there was a trend favoring the robot-trained group. Although these findings are modest, they provided unequivocal evidence that movement therapy of the kind that might be delivered by a robot had a significant positive impact on recovery (Aisen et al., 1997; Volpe et al., 1999, 2000; Krebs et al., 2000). 
Ninety-six stroke survivors were recalled and a third of them re-examined (Volpe et al., 2001). Most of these patients had received no therapy after discharge, yet two things were observed: first the robot group maintained the advantage over the control group and, second, both groups demonstrated greater reductions in impairment. This was contrary to the existing state of knowledge at the time which indicated that there were no further gains in motor abilities 3 months following stroke onset (Nakayama et al., 1994; Jorgensen et al., 1995). These results suggest that further improvement is possible in the chronic phase. Spurred on by these results, we initiated trials with chronic stroke in 2000.

\section{Chronic stroke}

Rather than examine our results with chronic patients (see, for example, Ferraro et al., 2003; Volpe et al., 2009), a publication in the New England Journal of Medicine is reviewed (Lo et al., 2010). This article presented the results of a multisite, independently run Veterans Affairs trial, CSP-558: ROBOTICS, on chronic stroke of upper extremity rehabilitation robotics employing the MIT-Manus for shoulder and elbow robot plus the corresponding anti-gravity, wrist, and hand robots (Lo et al., 2010) (Fig. 23.9 and Fig. 23.10). The publication was exciting for many reasons, not least because it was a rare publication on stroke rehabilitation published by this journal; we found only one other paper on rehabilitation following a stroke (Brashear et al., 2002).

CSP-558:ROBOTICS had an unusual design and it was comparable to a mixture of phase 2 and phase 3 studies. It evaluated the safety of these rehabilitation robots - they passed with flying colors. There were no serious adverse events in the robot group. A few patients mentioned muscle soreness, which is not surprising considering that they were making 1024 movements in an hour-long robot session with the paretic limb (instead of the typical 45 movements in "usual care" for chronic patients). The study also evaluated efficacy and cost. The first and perhaps most understated finding of CSP-558:ROBOTICS was that usual care (three sessions per week from therapists delivering treatment as they saw fit for the upper extremity) did not reduce impairment, disability, or improve quality of life in chronic stroke survivors. The usual care intervention had no measurable impact and was discontinued midway through the study. While it is possible that usual care prevents further decline, we believe that delivering three therapy sessions per week for the upper extremity should achieve some level of improvement beyond simple maintenance of the status-quo.

The trial lasted for approximately another year and compared robotic training for the shoulder and elbow, wrist, and hand that delivered 1024 movements three times per week with an intensive comparison training (ICT) protocol that we created to generate a positive control, in which a therapist delivered comparable movement intensity and repetition during the same period (Volpe et al., 2008). We expected the positive control to offer an advantage over usual care, due to the intense movement training that required the patient to actively use the paralyzed limb for the 50-minute session. We also expected the two experimental interventions to have a comparable effect, as demonstrated in the pilot study (Volpe et al., 2008), and this was borne out by the ROBOTICS results: there was no difference between the robotic and intensive therapy training group in motor outcome measures. However, a note of caution is required because this intervention is not conventional therapy, being very 
labor intensive and impractical to implement as standard care in a clinic. We were able to implement this intervention because, in most cases, the Veterans Affairs (VA) therapists delivering this form of training were engaged in ICT on average only once per day.

There are additional important considerations that need to be highlighted: (a) the comparison between the robot group and usual care involved roughly only the first half of the study, and (b) we were interested not only in the immediate or 12-week impact, but also whether the changes were robust and long lasting. On this score, robot therapy was statistically superior to usual care in Stroke Impact Scale (quality of life) at the completion of the intervention and also in the Fugl-Meyer (impairment) and Wolf Motor Function (function) 6 months following the completion of the intervention (Wolf et al., 1989; Duncan et al., 2003). The results are far more impressive if we compare the whole robot group with the usual care and not just the analysis that focused on the first half of the study. The results at 12 weeks show that the difference between the first half of the robotic treatment group and usual care was slightly over 2 Fugl-Meyer points. However, the difference between subjects receiving robotic treatment in the second half of the study and subjects receiving usual care during the first half of the study was almost 8 points in the Fugl-Meyer assessment (the total robotic group versus the total usual care showed a 5-point change).

It is quite important to stress that these groups of patients with chronic stroke disability were moderately to severely impaired (admission motor impairment scales averaged 19 out of a total score of 66) and over $30 \%$ had multiple strokes. As such, the group represented a spectrum of disability burden that many studies have avoided and, in our case, represented the majority of the cases (65\% of the volunteers enrolled). Thus, even though the improvements in the robotic therapy group might appear modest, the persistent improvement at the 6-month follow-up suggests increased robustness and perhaps an incremental advantage that prompted further recovery even in the absence of additional intervention. For example, an improvement of roughly 3 points in the Fugl-Meyer scale would enable a very severely affected patient to raise the arm and bathe independently, or stretch the formerly paralyzed arm so that independent dressing could take place. It might enable a more moderately affected stroke patient to tuck in their shirt or hike their pants independently, or reach overhead and actively grasp an object.

In this era of cost constraints, an important result was supplied by a cost-benefit analysis. While the active interventions added cost (for example, the added cost of the robotic equipment and the expense of an additional therapist cost the VA $\$ 10000$ per patient for 36 months), when we compared total cost, which included all the clinical care needed to take care of these veterans, there were no differences between active intervention and usual care. Total cost for the usual care group was roughly the same $(\$ 15000)$ because that group used the health-care system for clinical care three times more often than the active intervention groups. In other words, for 36 weeks of care the robotic group cost the VA $\$ 10000$ for robotic therapy and $\$ 5000$ for clinical care; 36 weeks of care in the usual care group cost the VA approximately $\$ 15000$. This suggests better care for the same total cost in the robotic group, but a full cost analysis is required before any firm conclusions can be drawn. 
In summary, we believe that robotic therapy that involves an interactive high-intensity, intention-driven therapy based on motor learning principles and assist-as-needed leads to better outcomes than usual care in chronic stroke (and probably with even greater impact in acute/subacute stroke). On September 28, 2010 the American Heart Association (AHA) issued "The Comprehensive Overview of Nursing and Interdisciplinary Rehabilitation Care of the Stroke Patient: A Scientific Statement from the American Heart Association" (Miller et al., 2010). It recommended that: "Robot-assisted therapy offers the amount of motor practice needed to relearn motor skills with less therapist assistance. Most robots for motor rehabilitation not only allow for robot assistance in movement initiation and guidance but also provide accurate feedback; some robots additionally provide movement resistance. Most trials of robot-assisted motor rehabilitation concern the upper extremity (UE), with robotics for the lower extremity (LE) still in its infancy. . Robot-assisted UE therapy, however, can improve motor function during the inpatient period after stroke.” The AHA suggested that robot-assisted therapy for the upper extremity has already achieved Class I, Level of Evidence A for Stroke Care in the Outpatient Setting and Care in Chronic Care Settings. It suggested that robot-assisted therapy for the upper extremity has achieved Class IIa, Level of Evidence A for Stroke Care in the Inpatient Setting. Class I is defined as: "Benefit $\gg$ Risk. Procedure/Treatment SHOULD be performed/administered"; Class IIa is defined as: "Benefit $\gg$ Risk, IT IS REASONABLE to perform procedure/administer treatment"; Level A is defined as "Multiple populations evaluated: Data derived from multiple randomized clinical trials or meta-analysis." The 2010 Veterans Administration/ Department of Defense guidelines for stroke care came to the same conclusion endorsing the use of rehabilitation robots for the upper extremity, but not yet for the lowerextremity(UnitedStatesDepartmentofVeteransAffairs, 2010).

Unfortunately we still do not know how to tailor therapy to a particular patient's needs. We do not know the optimal dose or in cost-benefit terms. What is the minimum intensity to promote actual change? Is too much therapy detrimental? Should we deliver impairmentbased or functionally based approaches and to whom: severe, moderate, or mild strokes? Should we deliver bi-manual or unilateral approaches and to whom? Should therapy progress proximal to distal or the other way around? Should we assist-as-needed, resist, or perturb and augment error? Who might be the responders who benefit most from these interventions? How should we integrate robotic training in therapy practices? The challenge for the next 10 years is to focus on the multitude of variables that may influence outcome, and to determine the interaction or independence among these variables and their actual impact on outcomes. Ultimately the goal should be determining how best to customize the treatment protocol to meet each individual patient's needs.

\section{Acknowledgments}

This work was supported in part by NIH grant \#1 R01-HD045343 and the Veterans Administration Baltimore Medical Center "Center of Excellence on Task-Oriented Exercise and Robotics in Neurological Diseases": B3688R. Dr. H. I. Krebs is a co-inventor of several MIT-held patents for robotic technology. He holds equity positions in Interactive Motion Technologies, the company that manufactures this type of technology under license to MIT. 


\section{References}

Aisen ML, Krebs HI, Hogan N, et al. The effect of robot-assisted therapy and rehabilitative training on motor recovery following stroke. Arch Neurol. 1997; 54:443-446. [PubMed: 9109746]

American Heart Association. Heart Disease and Stroke Statistics - 2010 Update. 2010

Brashear A, Gordon MF, Elovi E, et al. Intramuscular injection of botulinun toxin for the treatment of wrist and finger spasticity after a stroke. N Engl J Med. 2002; 347:395-400. [PubMed: 12167681]

Butler P, Major R, Patrick J. The technique of reciprocal walking using the hip guidance orthosis (HGO) with crutches. Prosthet Orthot Int. 1984; 8:33-38. [PubMed: 6718236]

Duncan PW, Bode RK, Min Lai S. Analysis of a new stroke-specific outcome scale: the Stroke Impact Scale. Arch Phys Med Rehabil. 2003; 84:950-963. [PubMed: 12881816]

Ferguson KA, Polando G, Kobetic R, et al. Walking with a hybrid orthosis system. Spinal Cord. 1999; 37:800-804. [PubMed: 10578252]

Ferraro M, Palazzolo JJ, Krol J, et al. Robot aided sensorimotor arm training improves outcome in patients with chronic stroke. Neurology. 2003; 61:1604-1607. [PubMed: 14663051]

Fugl-Meyer AR, Jaasko L, Leyman I, et al. The post-stroke hemiplegic patient. 1. A method for evaluation of physical performance. Scand J Rehabil Med. 1975; 7:13-31. [PubMed: 1135616]

Goldfarb M, Durfee WK. Design of a controlled-brake orthosis for FES-aided gait. IEEE Trans Rehabil Eng. 1996; 4:13-24. [PubMed: 8798068]

Goldfarb M, Korkowski K, Harrold B, et al. Preliminary evaluation of a controlled-brake orthosis for FES-aided gait. IEEE Trans Neural Syst Rehabil Eng. 2003; 11:241-248. [PubMed: 14518787]

Greene PJ, Granat MH. A knee and ankle flexing hybrid orthosis for paraplegic ambulation. Med Eng Phys. 2003; 25:539-545. [PubMed: 12835066]

Hallett M. Plasticity in the human motor system. Neuroscientist. 1999; 5:324-332.

Hidler J, et al. Multicenter randomized clinical trial evaluating the effectiveness of the Lokomat in subacute stroke. Neurorehabil Neural Repair. 2009; 23:5-13. [PubMed: 19109447]

Hirokawa S, Grimm M, Le T, et al. Energy consumption in paraplegic ambulation using the reciprocating gait orthosis and electric stimulation of the thigh muscles. Arch Phys Med Rehabil. 1990; 71:687-694. [PubMed: 2375676]

Hornby TG, et al. Enhanced gait-related improvements after therapist- versus robotic-assisted locomotor training in subjects with chronic stroke: a randomized controlled study. Stroke. 2008; 39:1786-1792. [PubMed: 18467648]

IEEE. Special Issue: 50 Years of Robotics. IEEE Robotics and Automation Magazine. 2010; 17

Isakov E, Douglas R, Berns P. Ambulation using the reciprocating gait orthosis and functional electrical stimulation. Paraplegia. 1992; 30:239-245. [PubMed: 1625891]

Jefferson R, Whittle M. Performance of three walking orthoses for the paralysed: a case study using gait analysis. Prosthet Orthot Int. 1990; 14:103-110. [PubMed: 2095527]

Jenkins WM, Merzenich MM. Reorganization of neurocortical representations after brain injury: a neurophysiological model of the bases of recovery from stroke. Progress Brain Res. 1987; 71:249_ 266.

Jorgensen HS, Nakayama H, Raaschou HO, et al. Outcome and time course of recovery in stroke Part II: Time course of recovery. The Copenhagen Stroke Study. Arch Phys Med Rehabil. 1995; 76:406-412. [PubMed: 7741609]

Krebs HI, Hogan N, Aisen ML, et al. Robot-aided neurorehabilitation. IEEE Trans Rehabil Eng. 1998; 6:75-87. [PubMed: 9535526]

Krebs HI, Volpe BT, Aisen ML, et al. Increasing productivity and quality of care: robot-aided neurorehabilitation. VA J Rehab Res Dev. 2000; 37:639-652.

Krebs, HI.; Hogan, N.; Durfee, W., et al. Rehabilitation Robotics, Orthotics, and Prosthetics; Chapter 48. In: Selzer, ME.; Clarke, S.; Cohen, LG.; Duncan, PW.; Gage, FH., editors. Textbook of Neural Repair and Rehabilitation. Vol. Ch 48. Cambridge University Press; Cambridge: 2006.

Krebs D, Levy-Tzedek Fa, Rykman Z, et al. Paradigm shift: therapeutic robotics. IEEE-EMBS Magazine. 2008; 27:61-70. 
Kwakkel G, Kollen BJ, Krebs HI. Effects of Robot-assisted therapy on upper limb recovery after stroke: a systematic review. Neurorehabil Neural Repair. 2008; 22:111-121. [PubMed: 17876068]

Lo AC, Guarino PD, Richards LG, et al. Peduzzi. Robot-assisted therapy for long-term upper-limb impairment after stroke. N Engl J Med. 2010; 362:1772-1783. [PubMed: 20400552]

Major R, Stallard J, Rose G. The dynamics of walking using the hip guidance orthosis (HGO) with crutches. Prosthet Orthot Int. 1981; 5:19-22. [PubMed: 7279608]

Marsolais EB, Kobetic R, Polando G, et al. The Case Western Reserve University hybrid gait orthosis. J Spinal Cord Med. 2000; 23:100-108. [PubMed: 10914350]

McClelland M, Andrews B, Patrick J, et al. Augmentation of the Oswestry Parawalker orthosis by means of surface electrical stimulation: gait analysis of three patients. Paraplegia. 1987; 25:32-38. [PubMed: 3562054]

Mehrholz J, Werner C, Kugler J, et al. Electromechanical-assisted training for walking after stroke. Cochrane Database Syst Rev. 2007:CD006185. [PubMed: 17943893]

Miller, P. Orthoses for the pelvic and hip region. In: Nawoczenski, D.; Epler, M., editors. Orthotics in Functional Rehabilitation of the Lower Limb. Saunders; Philadelphia: 1997. p. 15-30.

Miller EL, Murray L, Richards L, et al. The Comprehensive Overview of Nursing and Interdisciplinary Rehabilitation Care of the Stroke Patient: A Scientific Statement from the American Heart Association. Stroke. 2010; 41:2402-2448. [PubMed: 20813995]

Nakayama H, Jorgensen HS, Raaschou HO, et al. Recovery of upper extremity function in stroke patients: the Copenhagen Stroke Study. Arch Phys Med Rehabil. 1994; 75:394-398. [PubMed: 8172497]

National Science Foundation. Emerging Frontiers in Research and Innovation. White Paper on NeuroRobotics: Mind, Machines, and Motor Control. http://www.nsf.gov/div/index.jsp?org=EFRI

Nef T, Mihelj M, Riener R. ARMin: a robot for patient-cooperative arm therapy. Med Biol Eng Comput. 2007; 45:887-900. [PubMed: 17674069]

Nene A, Jennings S. Hybrid paraplegic locomotion with the Parawalker using intramuscular stimulation: a single subject study. Paraplegia. 1989; 27:125-132. [PubMed: 2785668]

Petrofsky J, Smith J. Physiologic costs of computer-controlled walking in persons with paraplegia using a reciprocatinggait orthosis. Arch Phys Med Rehabil. 1991; 72:890-896. [PubMed: 1929807]

Popovic D. Dynamics of the self-fitting modular orthosis. IEEE Trans Robotics Automat. 1990; 6:200-207.

Popovic D, Tomovic R, Schwirtlich L. Hybrid assistive system: the motor neuroprosthesis. IEEE Trans Biomed Eng. 1989; 36:729-737. [PubMed: 2787281]

Popovic, D.; Schwirtlich, L.; Radosavljevic, S. Powered hybrid assistive system. In: Popovic, D., editor. Advances in External Control of Human Extremities. NAUKA; 1990. p. 177-186.

Riener R, Lunenburger L, Jezernik S, et al. Patient-cooperative strategies for robot-aided treadmill training: first experimental results. IEEE Trans Neural Syst Rehabil Eng. 2005; 13:380-394. [PubMed: 16200761]

Solomonow M, Baratta R, Hirokawa S, et al. The RGO generation II: muscle stimulation powered orthosis as a practical walking system for thoracic paraplegics. Orthopedics. 1989; 12:1309-1315. [PubMed: 2798239]

Solomonow M, Aguilar E, Reisin E, et al. Reciprocating gait orthosis powered with electrical muscle stimulation (RGO II). Part I: performance evaluation of 70 paraplegic patients. Orthopedics. 1997; 20:315-324. [PubMed: 9127865]

Stallard J, Major R, Patrick J. A review of the fundamental design problems of providing ambulation for paraplegic patients. Paraplegia. 1989; 27:70-75. [PubMed: 2784201]

Stein J, Narendran K, McBean J, et al. Electromyography-controlled exoskeletal upper-limb-powered orthosis for exercise training after stroke. Am J Phys Med Rehabil. 2007; 86

Tomovic R, Vukobrativic M, Vodovnik L. Hybrid actuators for orthotic systems: hybrid assistive systems. Advances in External Control of Human Extremities. 1973; IV:73-80.

United States Department of Veterans Affairs, Department of Defense, American Heart Association, American Stroke Association. VA/DoD clinical practice guideline for the management of stroke 
rehabilitation. The Office of Quality and Performance, VA, Washington, DC \& Quality Management Division, United States Army MEDCOM (Version 3.0). 2010. Full guideline available at: http://www.healthquality.va.gov or, http://www.qmo.amedd.army.mil

US Congressional Robotics Caucus. A Roadmap for Robotics: From Internet to Robotics. Chaired by Congressman Mike Doyle (PA) and and co-chaired by Congressman Phil Gingrey (GA). http:// www.roboticscaucus.org/

Volpe BT, Krebs HI, Hogan N, et al. Robot training enhanced motor outcome in patients with stroke maintained over 3 years. Neurology. 1999; 53:1874-1876. [PubMed: 10563646]

Volpe BT, Krebs HI, Hogan N, et al. A novel approach to stroke rehabilitation: robot aided sensorymotor stimulation. Neurology. 2000; 54:1938-1944. [PubMed: 10822433]

Volpe BT, Krebs HI, Hogan N. Is robot-aided sensori-motor training in stroke rehabilitation a realistic option? Curr Opin Neurol. 2001; 14:745-752. [PubMed: 11723383]

Volpe BT, Lynch D, Ferraro M, et al. Intensive sensorimotor arm training improves hemiparesis in patients with chronic stroke. Neurorehabil Neural Repair. 2008; 22:305-310. [PubMed: 18184932]

Volpe BT, Huerta PT, Zipse J, et al. Robotic devices as therapeutic and diagnostic tools for stroke recovery. Arch Neurol. 2009; 66:1086-1090. [PubMed: 19752297]

Weber DJ, Skidmore ER, Niyonkuru C, et al. Cyclic functional electrical stimulation does not enhance gains in hand grasp function when used as an adjunct to onabotulinumtoxina and task practice therapy: a single-blind, randomized controlled pilot study. Arch Phys Med Rehabil. 2010; 91:679686. [PubMed: 20434603]

Whittle M, Cochrane G, Chase A, et al. A comparative trial of two walking systems for paralysed people. Paraplegia. 1991; 29:97-102. [PubMed: 2023783]

Wolf SL, Lecraw DE, Barton LA, et al. Forced use of hemiplegic upper extremities to reverse the effect of learned nonuse among chronic stroke and head-injured patients. Exp Neurol. 1989; 104:125-132. [PubMed: 2707361]

Yang L, Granat MH, Paul JP, et al. Further development of hybrid functional electrical stimulation orthoses. Artif Organs. 1997; 21:183-187. [PubMed: 9148700] 


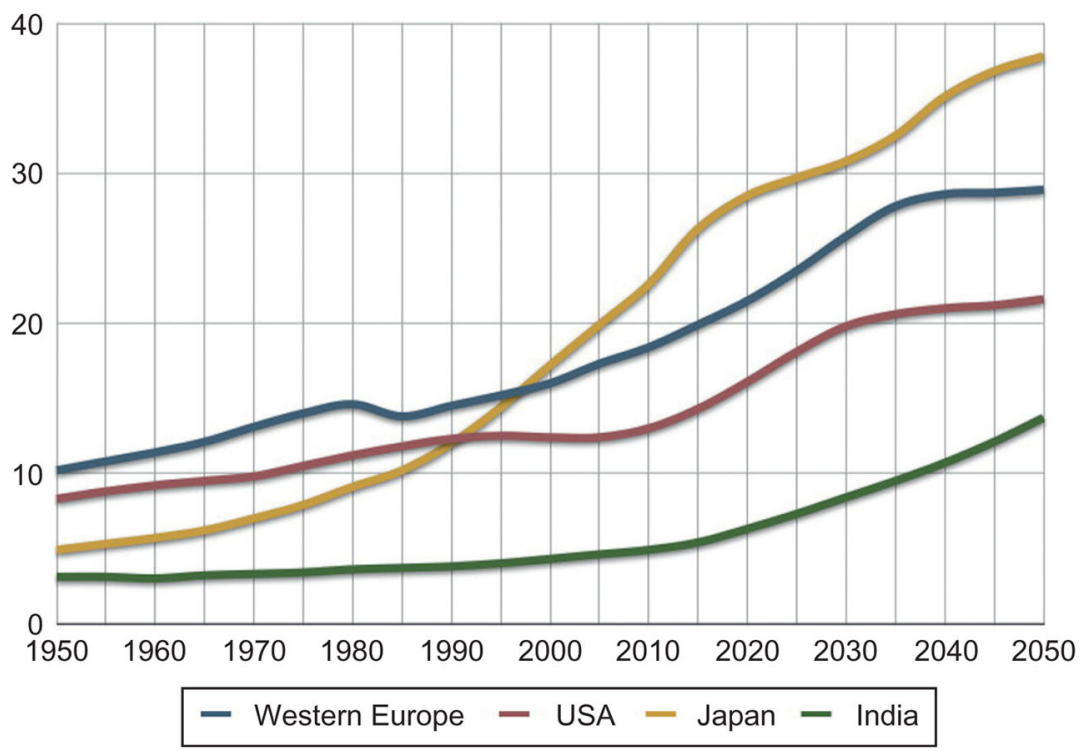

Fig. 23.1.

Percentage of population above 65 years of age (UN 2008 Data Series). (Courtesy of IEEE Robotics and Automation Magazine and Professor Henrik Christensen: IEEE, 2010.) 


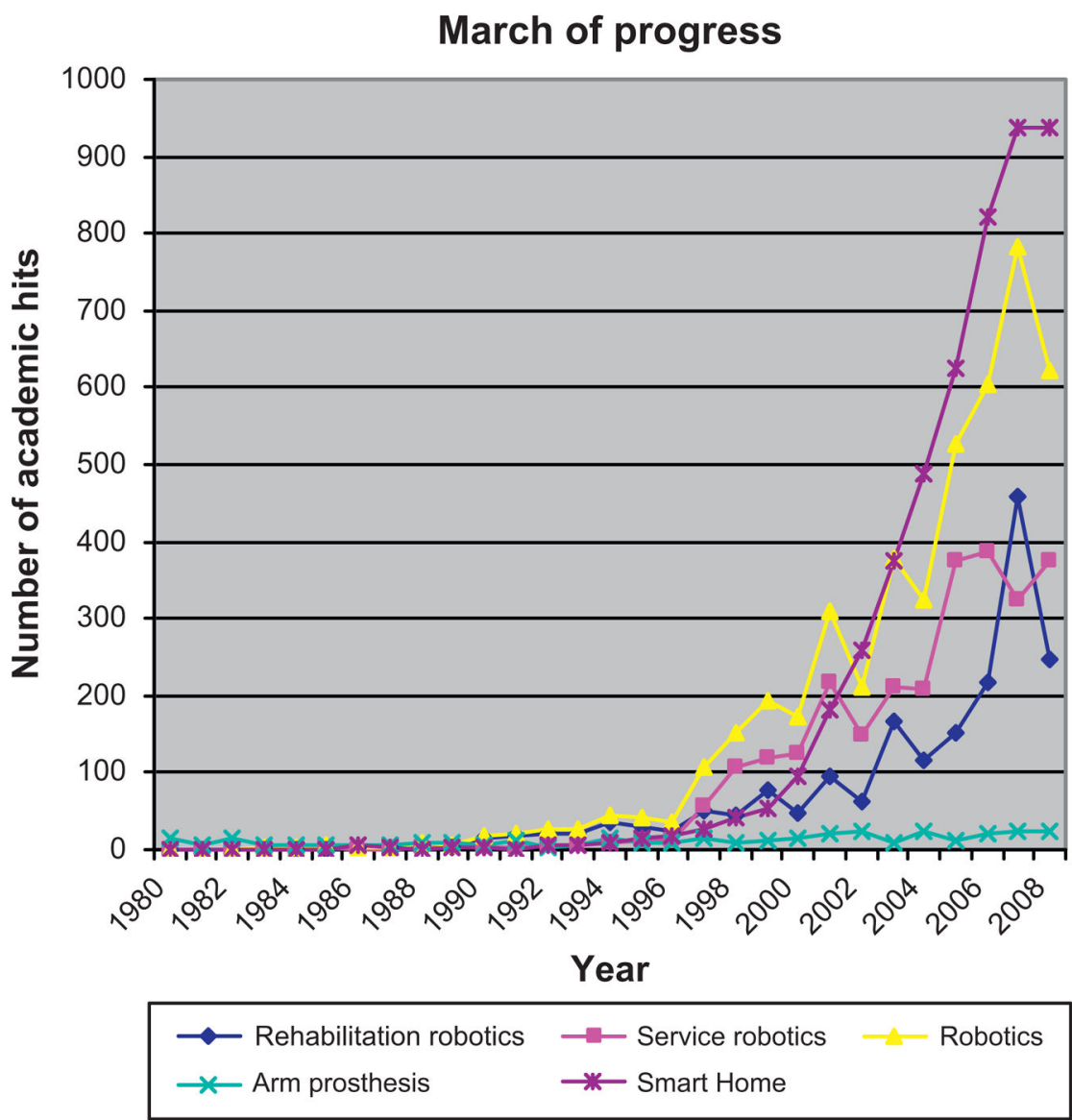

Fig. 23.2.

Number of hits of academic papers and keywords. 


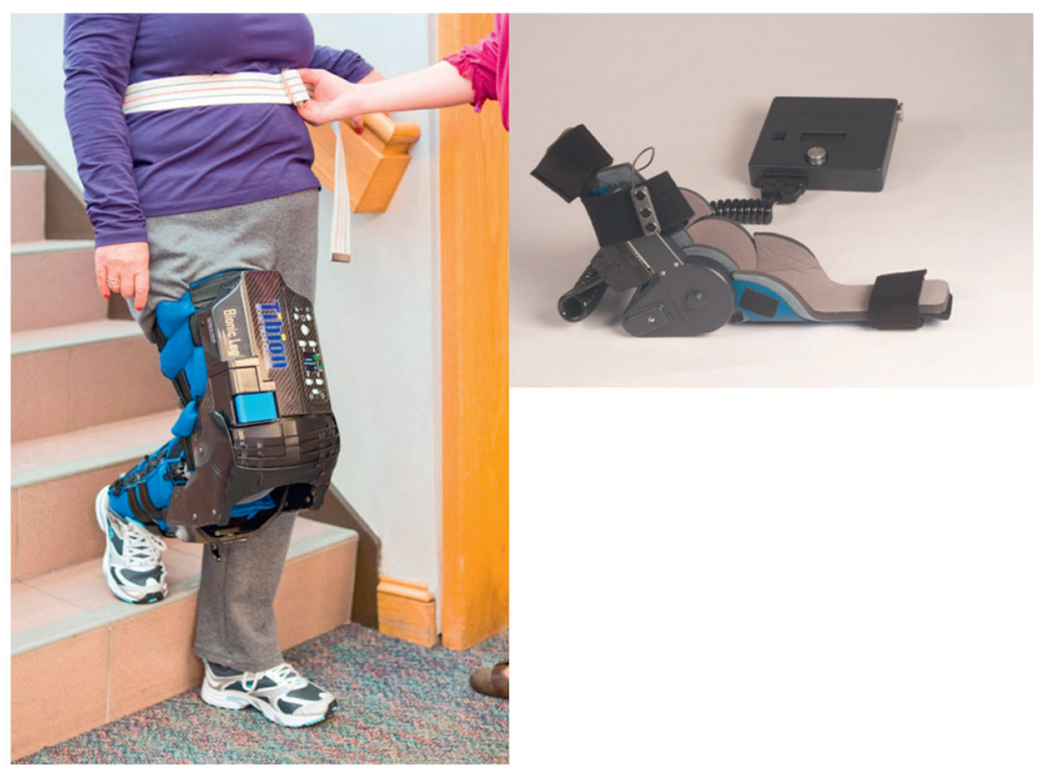

Fig. 23.3.

Myomo e100 and Tybion. They can actuate the elbow and knee of stroke patients. (Courtesy Tybion and J. Stein, Department of Rehabilitation and Regenerative Medicine, Columbia University and Division of Rehabilitation Medicine, Weill Cornell Medical College.) 


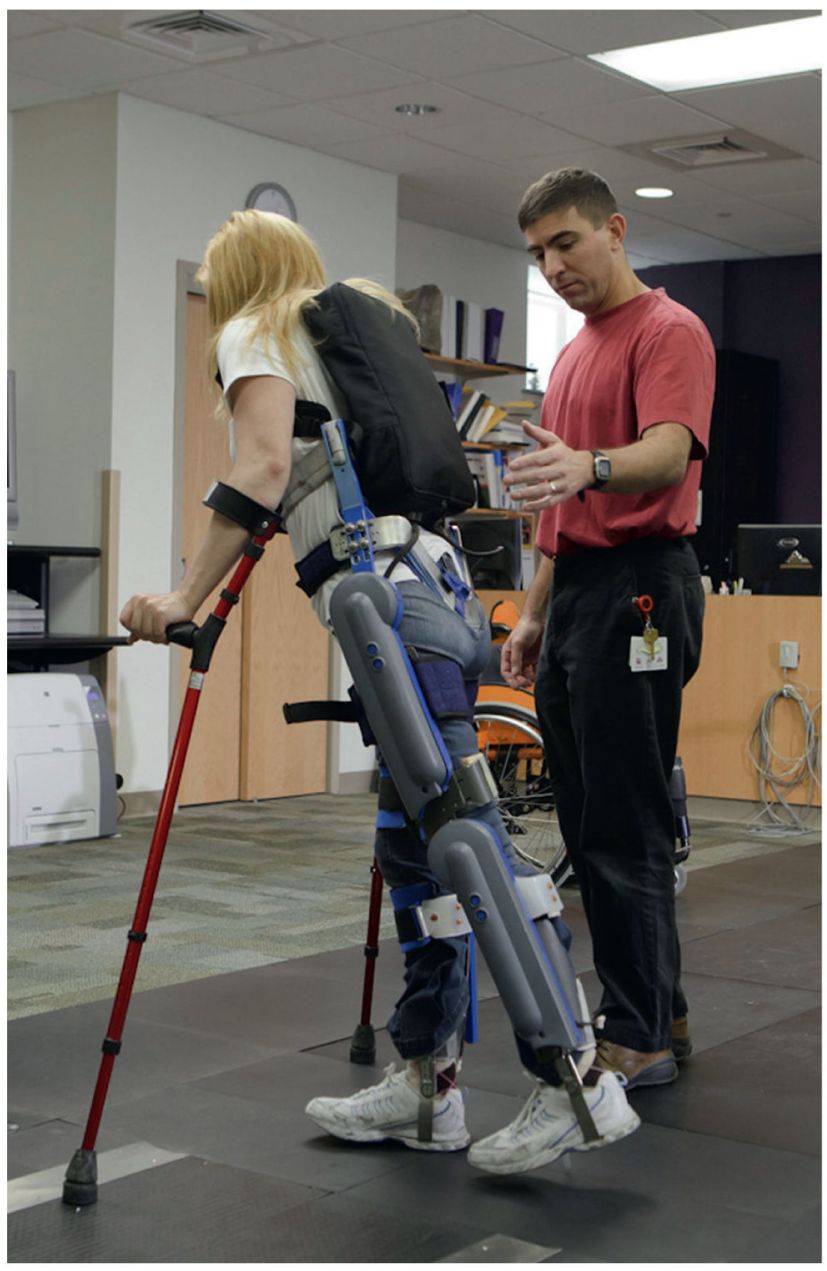

Fig. 23.4.

Re-Walk from Argo. (Courtesy of A. Esquenazi, Department of Physical Medicine and Rehabilitation, Moss Rehabilitation Hospital.) 

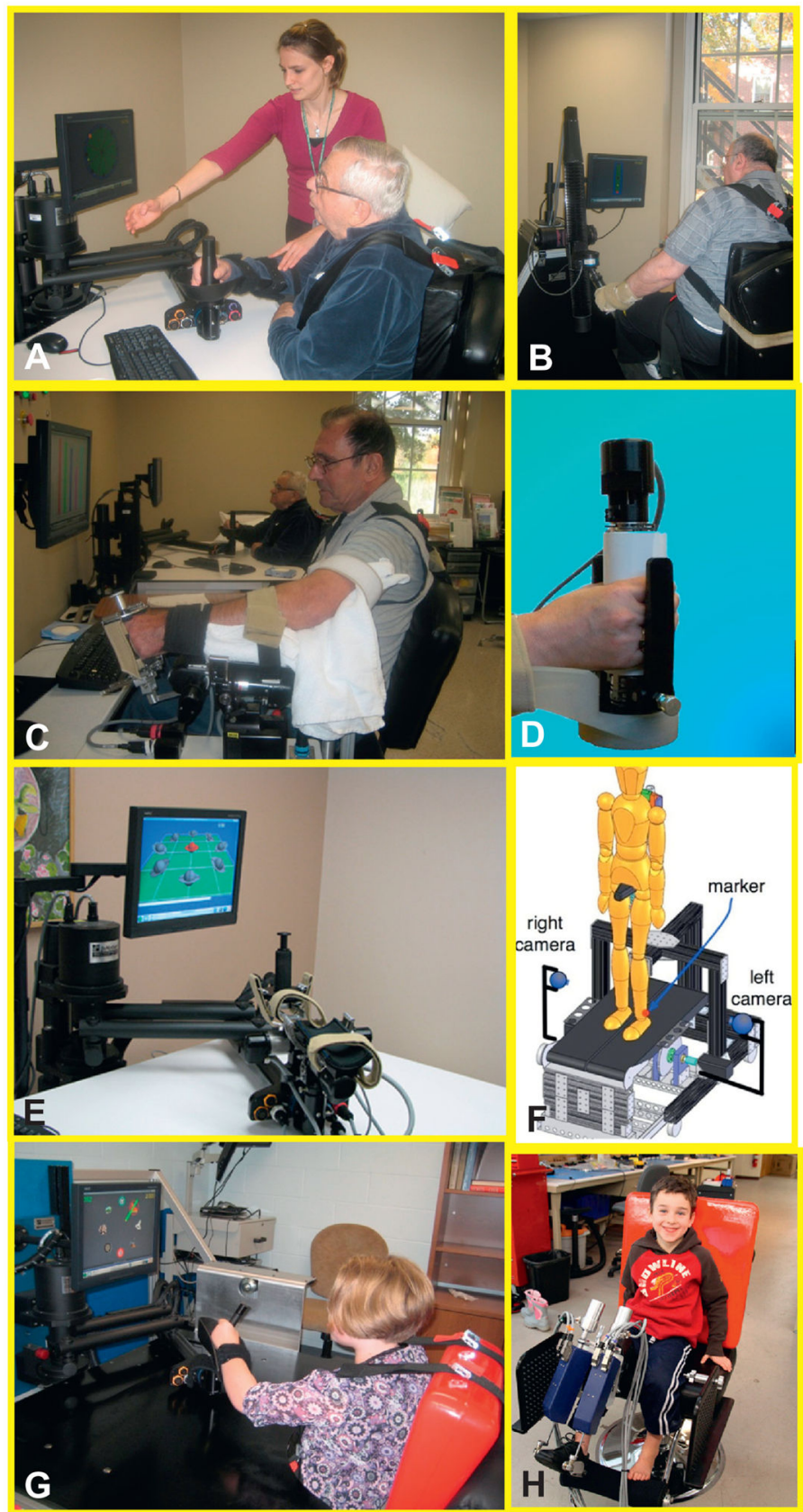

Fig. 23.5.

MIT Gym of Robots (commercialized by Interactive Motion Technologies, Watertown, MA). (A) MIT-Manus to promote neurorecovery of the injured brain and control of the shoulder and elbow segments; (B) the antigravity to promote training of the shoulder against gravity. (C) The wrist robot which affords training of the 3 degrees of freedom of the wrist and forearm; (D) the hand module for grasp and release. (E) The combination of shoulder and elbow robot with the wrist module mounted at the tip of first affording training for both transport of arm and object manipulation; (F)a sketch of the alpha-prototype of the MIT- 
Skywalker for gait training. (G) Pediatric population working with the MIT-Manus and (H) our pediatric Anklebot that affords training in dorsi/plantarflexion and inversion/eversion. 


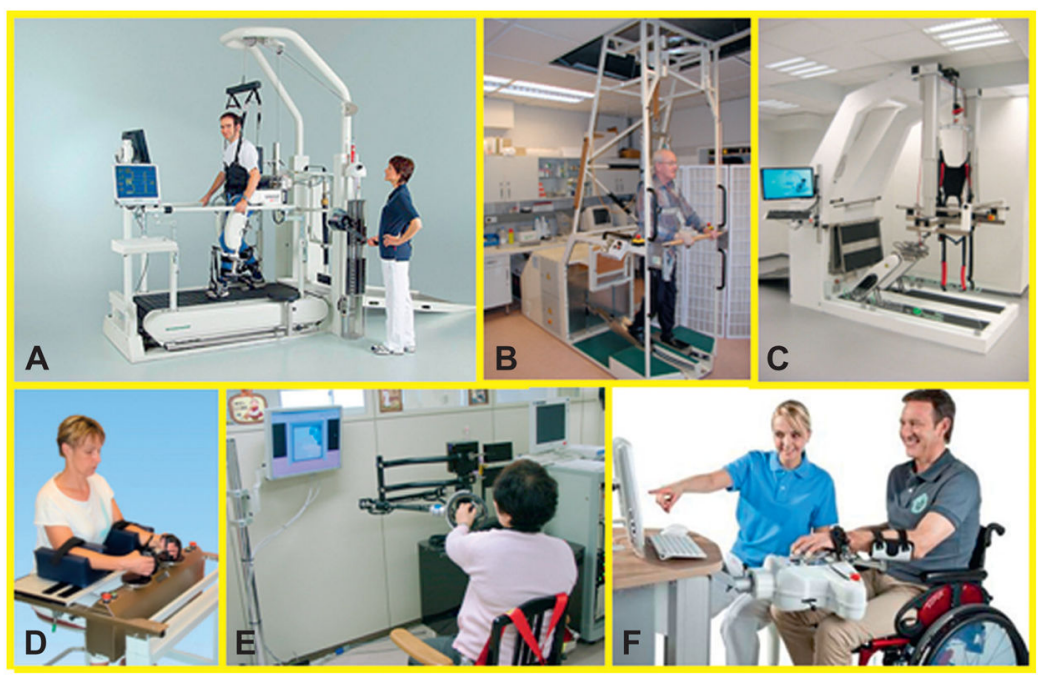

Fig. 23.6.

Examples of rehabilitation robots. (A) The Lokomat (Hocoma, Switzerland) which is an exoskeletal robot to manipulate patient's hip and knee. (B) The Gait Trainer I and (C) the GEO (Reha-Stim, Germany), which are end-effector robots that manipulate tje patient's foot. (D) The Bimanutrak for bimanual training of wrist and forearm (Reha-Stim, Germany). (E) Osaka University's shoulder and elbow robot (Asahi Chemical Industry) and (F) the Amadeo to manipulate the individual fingers (Tyromotion, Austria). 
Fugl-Meyer (mean and 95\% Cl)

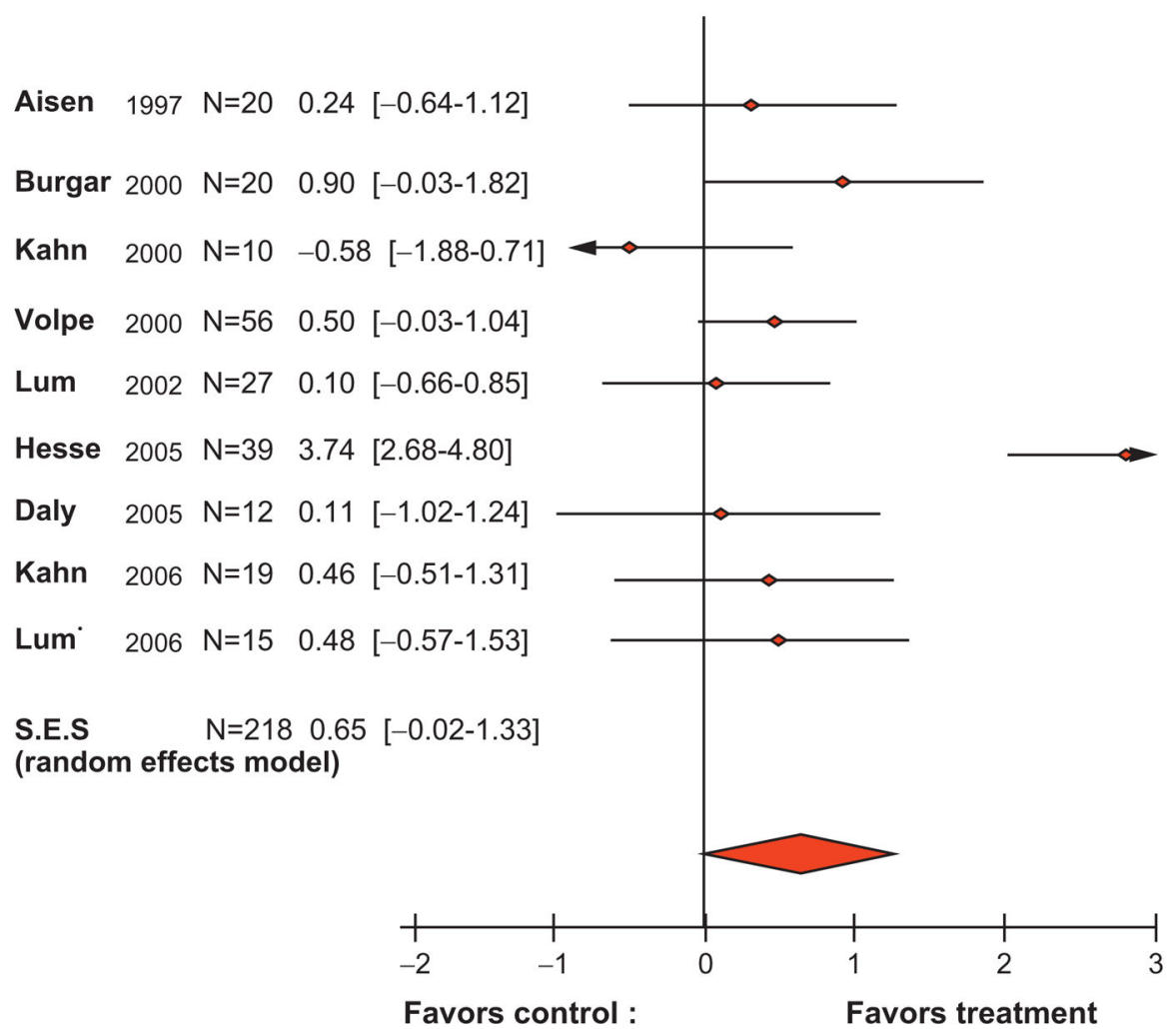

Fig. 23.7.

Meta-analysis of robot-assisted therapy trials on motor recovery following stroke. (Kwakkel et al., 2009). 


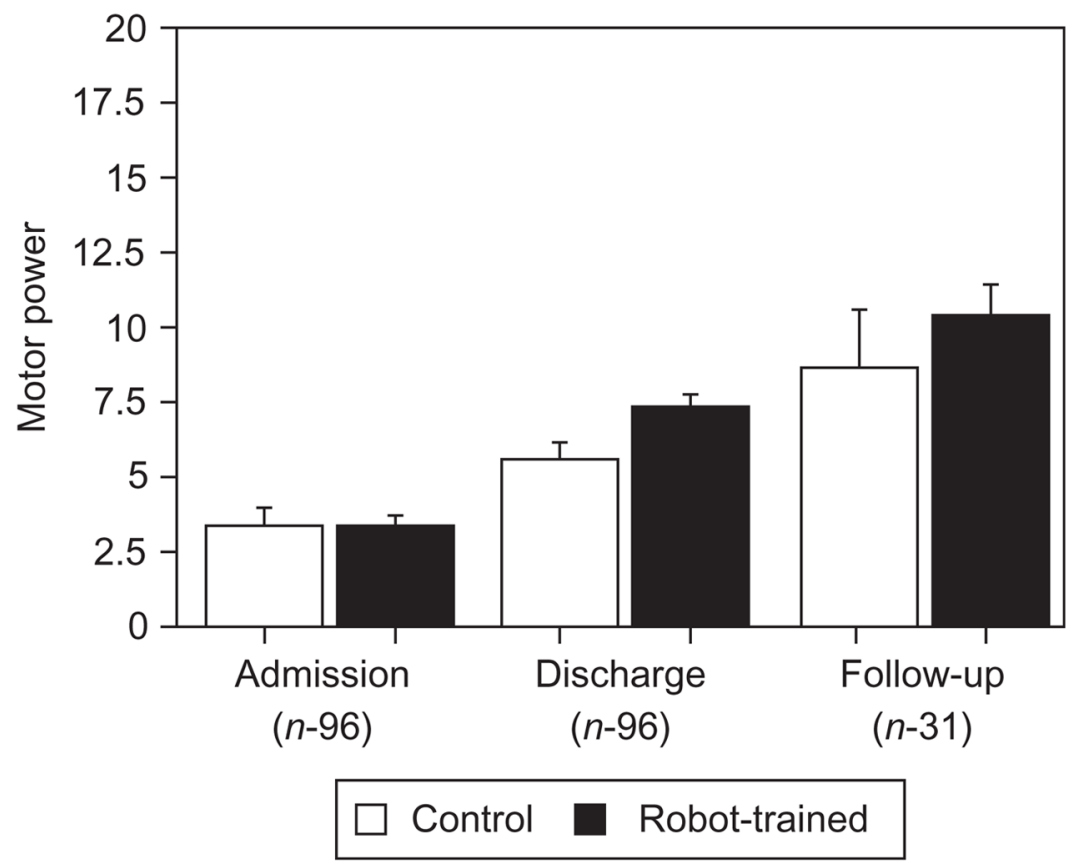

Fig. 23.8.

Burke inpatient studies $(\mathrm{N}=96)$. Mean interval change in the Motor Power Scale (Volpe et al., 2001) (significance $\mathrm{p}<0.05$ ). Mean \pm standard error Motor Power scores (maximum score 20) of 96 patients on admission before rehabilitation, at discharge after rehabilitation, and robotic training or control, and at follow-up evaluation approximately 3 years after stroke. Robot-trained patients maintain the motor improvements. ${ }^{*} \mathrm{P}<0.05$, versus control. 
Primary Outcome - Fugl - Meyer Change at 12 weeks

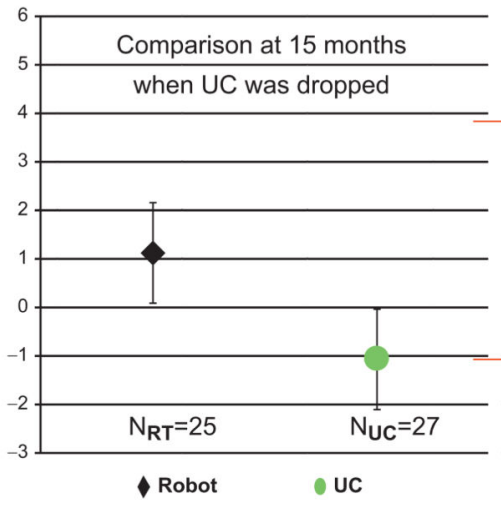

Mean Difference $=2.17, p=0.08$

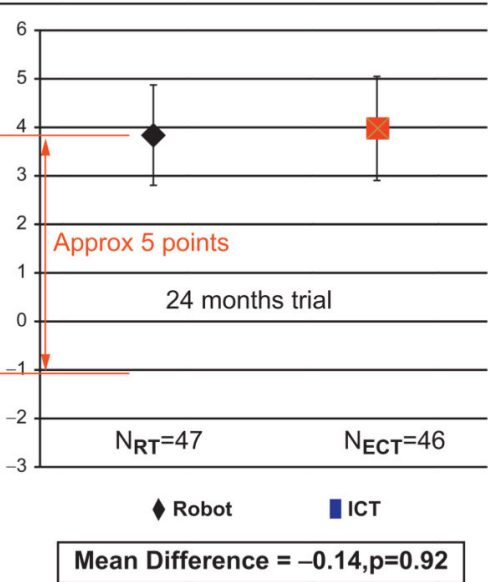

Mean Difference $=-0.14, p=0.92$

Fig. 23.9.

ROBOTICS (CSP-558) primary results at 12 weeks (therapy completion). Left panel shows the changes in the primary outcome for the robot and usual care groups during the initial half of the trial. Right panel shows the changes in the primary outcome for the robot and intensive comparison training groups during the whole duration of the trial. Red vertical arrow indicates the change in the primary outcome of the complete robot group in relation to the usual care group. 

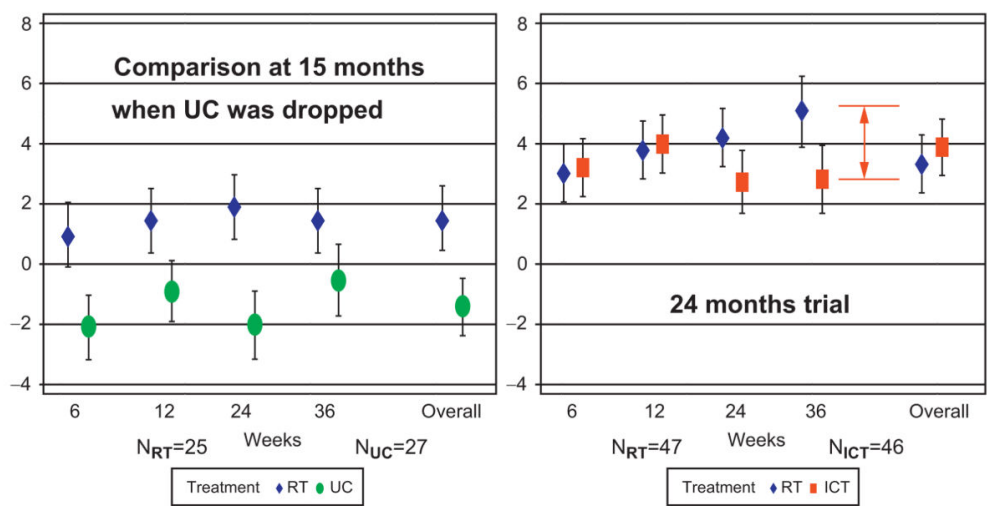

Overall Mean Difference over 36 weeks Robot vs. UC $=2.88, p=.016$

Overall Mean Difference over 36 weeks Robot vs. ICT $=-0.58, \mathrm{p}=.63$

Fig. 23.10.

ROBOTICS (CSP-558) results at 36 weeks (after 6 months follow-up). The figure shows the changes in the primary outcome over the duration of the intervention (evaluations at 6 and 12 weeks) and during the 6-month follow-up period (evaluations at 24 and 36 weeks). Both panels also show the estimated changes at 36 weeks using a fixed-model to fit all the data (overall). Left panel shows results for robot and usual care groups during the initial half of the study. Right panel shows results for the robot and intensive comparison training groups during the whole duration of the trial. Note that the robot group continues to improve after the intervention is completed (see evaluations at 24 and 36 weeks). Red vertical arrow indicates the actual change in the primary outcome of the complete robot group in relation to the intensive comparison training group at 36 weeks (instead of the overall fixed-model estimate shown on the right). 